Revue de droit comparé du travail et de la sécurité sociale

3 | 2020

La Directive 2019/1158 du 20 juin 2019 concernant l'équilibre entre vie personnelle et vie privée des parents et des aidants

\title{
La Sécurité sociale au défi de la pandémie
}

Jean-Pierre Laborde

\section{(2) OpenEdition}

Journals

Édition électronique

URL : https://journals.openedition.org/rdctss/1037

DOI : $10.4000 /$ rdctss. 1037

ISSN : 2262-9815

Éditeur

Centre de droit comparé du travail et de la sécurité sociale

Édition imprimée

Date de publication : 1 novembre 2020

Pagination : 206-211

ISSN : $2117-4350$

Référence électronique

Jean-Pierre Laborde, "La Sécurité sociale au défi de la pandémie », Revue de droit comparé du travail et de la sécurité sociale [En ligne], 3 | 2020, mis en ligne le 01 novembre 2021, consulté le 11 novembre 2021. URL : http://journals.openedition.org/rdctss/1037 ; DOI : https://doi.org/10.4000/rdctss.1037

\section{(c) $(1) \Theta \Theta$}

Revue de droit comparé du travail et de la sécurité sociale est mise à disposition selon les termes de la Licence Creative Commons Attribution - Pas d'Utilisation Commerciale - Pas de Modification 4.0 International. 


\section{JEAN-PIERRE LABORDE}

Université de BordeAux, COMPtrasec UMR 5114- CNRS

\section{LA SÉCURITÉ SOCIALE AU DÉFI DE LA PANDÉMIE}

Le système français de sécurité sociale et de protection sociale a déjà connu toutes sortes de semestres. Le premier semestre de 2020 aura cependant été le plus étrange d'entre tous. Il en a été ainsi en raison de la pandémie qui a commencé à frapper la France à la fin de l'année 2019 et qui est allée rapidement s'aggravant dans le premier trimestre de 2020, et également en raison du confinement général instauré par les pouvoirs publics le 17 mars 2020 et prolongé jusqu'au 11 mai suivant, soit pendant près de huit semaines. Il faut en outre signaler que ce confinement a été suivi d'un " déconfinement » assez lentement progressif et encadré tout au long par une loi d'urgence sanitaire.

Au moment où nous écrivons ces lignes la « crise sanitaire » est du reste loin d'être complètement achevée.

Il est évident qu'une telle situation a eu - et continuera à avoir - un fort impact sur notre système de sécurité sociale. Même s'il est sans doute un peu trop tôt pour se hasarder aux bilans, on peut penser que le rôle qu'a joué le système de sécurité sociale a été important, aussi bien dans le traitement de la pandémie que dans l'accompagnement du confinement et, somme toute, tout à fait positif.

Pour autant, s'il convient de saluer l'efficacité des réponses immédiates (I), il faut aussi s'interroger, à plus long terme, sur les défis que le système de sécurité sociale risque de devoir affronter pour longtemps (II).

\section{I - LES RÉPONSES IMMÉDIATES}

Ces réponses ont concerné aussi bien la branche maladie (A) que la branche retraite (B) et, hors du système proprement dit de Sécurité sociale, l'indemnisation du chômage (C).

\section{A - LA BRANCHE MALADIE}

S'agissant de la prise en charge des dépenses de santé, la couverture générale s'est évidemment étendue à tous les soins liés à l'épidémie de coronavirus, que ces soins aient été de prévention, de prospection ou de traitement. Exceptionnellement, cette couverture n'a été réduite par aucun ticket modérateur. Bien entendu, toujours dans la branche maladie, les prestations en espèces ont été servies à toutes celles et ceux que la survenance de la pandémie a contraint à un ou plusieurs arrêts de travail. 
Il faut ajouter ici que, dans un souci d'assurer une couverture aussi complète que possible, les indemnités journalières ont couvert, sans franchise, l'intégralité des arrêts de travail dès leur première journée (article 8 de la loi 2020-290 du 23 mars 2020 d'urgence pour faire face à l'épidémie de Covid-19).

Pour généreuse qu'elle ait été, cette disposition temporaire - qui a cessé le 11 juillet 2020 avec l'état d'urgence lui-même - était attendue dans la mesure où, en période de grave épidémie, il eût été mal venu d'appliquer aux victimes une franchise qui tend à sanctionner une propension trop grande à l'absentéisme au travail. On mesure ici qu'il est des circonstances où le souci de la responsabilisation des assurés sociaux va dans le sens d'une plus franche indemnisation, plutôt que dans celui de la lutte contre les comportements suspects. II serait ainsi tout à fait absurde d'inciter des salariés potentiellement malades à prolonger de quelques jours leur maintien au travail.

Le législateur est allé encore plus loin dans le sens d'une extension pure et simple de la couverture, même si celle-ci a bien sûr été temporaire.

Deux décrets du 31 janvier 2020 et du 9 mars 2020 ont étendu le service des indemnités journalières de l'assurance maladie aux salariés et aux travailleurs indépendants contraints de cesser leur travail pour assurer la garde de leurs enfants et sans possibilité de télétravail, ou en raison de mesures de quarantaine les affectant notamment parce qu'ils avaient été en contact avec une personne malade ou de mesures semblables concernant un enfant mineur de 16 ans. Où l'on voit que le risque couvert a ici été plus souplement entendu qu'en période ordinaire, dans une conception plus largement synthétique que strictement analytique de la couverture sociale.

Pareil souci d'extension a-t-il concerné - ou peut-il concerner - la couverture des risques professionnels, accidents du travail et maladies professionnelles?

Plus que d'une extension véritable, il s'agit davantage dans cette branche particulière d'un assouplissement, plus spécialement d'un allongement des délais de procédure pour ce qui concerne la déclaration, l'instruction et le contentieux des accidents du travail et des maladies professionnels (AT-MP). On remarquera que, selon les cas, ces assouplissements peuvent être favorables aux employeurs comme aux victimes.

II faut aussi souligner que les dispositions ici évoquées, fixées par trois Ordonnances des 25 mars, 22 avril et 17 juin 2020, ont été applicables dès le 12 mars 2020, soit avec rétroactivité, ce qui aura conduit les caisses à devoir s'adapter à un dispositif qu'elles n'ont pas connu dès le début de son application. Bien sûr ces dispositions sont temporaires, jusqu'à un arrêté du ministre chargé de la sécurité sociale, qui ne pourra en principe être postérieur à l'automne 2020.

L'assouplissement des dispositions n'est cependant pas destiné à s'arrêter là. Il va également concerner la reconnaissance du Covid comme maladie professionnelle alors que celui-ci ne figure pas pour l'heure dans les tableaux de maladies professionnelles. 
Cette reconnaissance simplifiée concerne les personnels soignants exposés au Covid, ainsi que les travailleurs non soignants qui ont dû poursuivre un travail en présentiel pendant la période de confinement.

Dans le même temps, pour ne pas pénaliser à l'excès les employeurs concernés, le financement des prestations servies aux victimes sera assez largement mutualisé.

\section{B - LA BRANCHE RETRAITE}

S'agissant des retraites, les périodes d'activité partielle ou, autrement dit, de chômage partiel, ont été exceptionnellement prises en compte pour le calcul des droits à retraite, alors pourtant qu'elles ne s'accompagnent d'aucune cotisation correspondante. L'urgence sociale a également amené le Gouvernement à suspendre la procédure législative de réforme du régime des retraites qui, dans un contexte de très vive contestation sociale, avait été engagée peu avant le début de la pandémie.

Au sortir du confinement, on pouvait imaginer, ou craindre, que le pouvoir exécutif veuille reprendre sans plus tarder le cours des réformes, y compris des réformes le plus contestées jusque-là.

II n'en aura finalement pas été ainsi puisque, après une période d'hésitation, le nouveau Premier Ministre, Monsieur Jean Castex, a annoncé, rencontrant ici l'assentiment des organisations syndicales, que la concertation avec les partenaires sociaux sur la réforme des retraites et sur celle de l'assurance chômage serait reportée de plusieurs mois.

\section{C - L'INDEMNISATION DU CHÔMAGE}

En effet, la souplesse a également concerné l'indemnisation du chômage, pourtant promise avant la pandémie à une réforme rigoureuse. Comme pour les retraites, cette réforme a été temporairement suspendue et en attendant les durées d'indemnisation, de même que les obligations de comportement attendues des demandeurs d'emploi, ont été - temporairement aussi - assouplies.

S'est ainsi mise en place une série de dispositifs de protection des salariés et des vulnérables, qui a quelques ressemblances avec les législations sociales des débuts de conflits armés, ce qui n'est pas très étonnant dans la mesure où la pandémie a été vécue comme une sorte d'agression généralisée.

C'est le fameux « Nous sommes en guerre », plusieurs fois répété par le Président Macron dans le discours qui a précédé la mise en place du confinement général,le Président Macron ayant par ailleurs ajouté qu'il en coûterait ce que cela doit coûter. Nul doute que tel est bien le cas de la Sécurité sociale.

\section{II - LES DÉFIS ET LES PERSPECTIVES}

Ces défis et perspectives concernent bien sûr, au premier chef, le financement de la sécurité sociale (A), mais aussi, et de manière moins attendue, la qualité et la solidité de la couverture sociale (B). 


\section{FRANCE}

\section{A - LE FINANCEMENT DE LA SÉCURITÉ SOCIALE}

II n'est aucunement besoin de beaucoup anticiper pour comprendre que l'un des résultats les plus manifestes de la crise sanitaire sera de mettre gravement à mal les finances de notre protection sociale en général, et de notre système de sécurité sociale en particulier. Vient en effet le temps de déficits qui, pris en valeur absolue, peuvent paraître abyssaux.

C'est en tout cas en milliards d'euros, et plus souvent encore en dizaines de milliards d'euros, qu'ils se chiffrent, et cela, dans toutes les composantes de notre couverture sociale, qu'il s'agisse de l'assurance maladie, de l'assurance retraite, de l'indemnisation du chômage.

Il est vrai que c'est aussi en dizaines, voire en centaines de milliards d'euros, que se compte traditionnellement un budget de la sécurité sociale dont il faut rappeler ici qu'il dépasse le budget de l'Etat.

Il est vrai encore que la différence proprement stupéfiante entre ces sommes qui paraissent défier l'entendement et la modestie de nombre de prestations effectivement versées aux individus, tient naturellement à ce que la couverture sociale concerne la quasi-totalité de la population dans la quasi-totalité de ses dépenses et besoins en matière de santé et de ressources. II n'en demeure pas moins que les réponses à la crise sanitaire actuelle passent par une accélération dangereuse des déficits sociaux.

Cette accélération est d'autant plus préoccupante que, contrairement à une idée trop simple pour être parfaitement exacte, elle est moins due à un excès des dépenses qu'à un tarissement des ressources.

La dégradation de la situation financière de la Sécurité sociale viendra certes pour partie d'un accroissement des dépenses, particulièrement dans la branche maladie. Mais elle viendra aussi et sans doute surtout d'une compression des recettes, liée aussi bien au ralentissement subit de l'activité économique - dans des conditions très peu connues jusque-là en temps de paix - qu'aux multiples dispositifs d'exonération, de suspension et de report des cotisations sociales, mis en place dans le souci par ailleurs bien compréhensible de donner un nouveau souffle aux entreprises et de meilleures chances à une reprise aussi indispensable qu'incertaine.

En mars dernier, le Président de la République avait été très clair en annonçant que tous les efforts seraient consentis, quoiqu'il en coûte, aussi bien dans la lutte contre la pandémie que dans la couverture des conséquences économiques et sociales du confinement. L'heure approche de l'estimation de ces coûts et ils seront sans nul doute impressionnants, en matière de Sécurité sociale comme ailleurs.

Or il faut rappeler qu'en dépit d'une politique sévère et parfois fort contestée - notamment en matière hospitalière - de contraction des coûts, la Sécurité sociale restait assez loin d'une position financière d'équilibre avant même l'irruption de la crise sanitaire. 
Les choses n'ont donc pu que s'aggraver et elles continueront de le faire tant que la situation sanitaire et économique et économique ne se sera pas purement et simplement inversée.

On peut dès lors imaginer, ou craindre, une nouvelle avancée de la fiscalisation du financement de la Sécurité sociale. Certes, les cotisations représentent toujours pour l'heure la majorité du financement, mais l'impôt - à $40 \%$ de ce financement - n'est plus si loin désormais et il ne fait guère de doute que sa part va encore augmenter. Comme on s'en doute, il ne s'agit pas seulement ici d'une question de chiffres et de nombres mais de responsabilités et de poids politiques, dans un système de Sécurité sociale dont l'autonomie vis-à-vis de l'Etat est de plus en plus strictement réduite.

\section{B - LA QUALITÉ ET LA SOLIDITÉ DE LA COUVERTURE SOCIALE}

On pourrait craindre qu'un horizon de financement aussi menaçant dissuade toute volonté d'amélioration ou d'extension de la couverture sociale. Or, de façon assez surprenante, c'est bien une réforme au moins en apparence très ambitieuse qui est annoncée, avec la création d'un « cinquième risque » de sécurité sociale, pour garantir une couverture efficace et pérenne de tout ce qui a trait à la dépendance, notamment des personnes âgées quand celles-ci ne peuvent effectuer sans soutien les gestes essentiels de la vie quotidienne.

Qui plus est, ce n'est pas seulement sur le terrain des prévisions que cette couverture de la dépendance est annoncée, mais dans la loi elle-même.

En effet, l'article 5 III de la loi du 7 août 2020 relative à la dette sociale et à l'autonomie dispose que «Au plus tard le 15 septembre 2020, le Gouvernement remet au Parlement, après consultation des différents financeurs, des collectivités territoriales ainsi que des associations de retraités et de personnes en situation de handicap et de représentants d'usagers et d'aidants, un rapport sur les modalités de mise en œuvre d'un nouveau risque et d'une nouvelle branche de la sécurité sociale relatifs au soutien à l'autonomie des personnes âgées et des personnes en situation de handicap ».

Ce même article ajoute que «Ce rapport présente les conséquences de la création de cette branche en termes d'architecture juridique et financière et en termes de pilotage, gouvernance et gestion de ce nouveau risque. »

Nouveau risque et nouvelle branche de la sécurité sociale : les termes assurément sont forts et le calendrier paraît devoir être serré.

Il est vrai que tout n'est pas à faire en matière d'autonomie puisqu'il existe déjà, notamment, une allocation personnalisée d'autonomie. Il est vrai que les dispositions de la loi du 7 août dernier annoncent une réforme à venir beaucoup plus qu'elles ne la mettent en œuvre. Et il vrai aussi que les arbitrages financiers, qui ne seront pas minces, ne sont pas encore arrêtés. Affaire donc à suivre.

Pour autant on ne peut manquer d'observer que ce coup d'accélérateur dans la couverture de la dépendance vise particulièrement à répondre aux situations les plus difficiles d'une catégorie de la population qui s'est révélée particulièrement 


\section{FRANCE}

vulnérable à l'emprise de la pandémie, celle du grand âge. Il est du reste fort souhaitable que l'on ne tarde pas trop à tirer toutes les leçons, nombreuses et diverses, de la lutte contre la pandémie. L'une de celles-ci est qu'il faudrait être plus que jamais attentif à tout ce qui concerne, en matière d'âge mais aussi, plus largement encore en matière de santé, la prévention et non plus seulement le traitement des maladies et des handicaps.

La prévention est très étroitement affaire d'information individuelle et collective et de responsabilité individuelle et collective. C'est un vaste champ d'intervention pour notre système de sécurité sociale. 